\title{
ALGUNAS DIFICULTADES EN TORNO A LA CONSERVACIÓN DE LA ENERGÍA
}

\author{
SOLBES, J. ${ }^{1}$ y TARÍN, F. ${ }^{2}$ \\ ${ }^{1}$ IB J. Rodrigo Botet. Santos Justo y Pastor, 70. 46940 Manises (Valencia). \\ ${ }^{2}$ IES de Picassent. Valencia.
}

\section{SUMMARY}

The subject of energy is a very important part of a physics course, as it provides a clear link between the different sections of physics. In this paper we analyze the teaching and learning of energy at secondary level and we show that traditional methods to teach energy: $a$ ) do not consider students misconceptions, $b$ ) introduce energy conservation in mechanics and thermodynamics but do not clarify if it is a theorem or a principle, and $c$ ) do not present the conservation of energy as a general principle of the whole physics. Finally, a new approach to energy teaching is showed.

\section{INTRODUCCIÓN}

Queremos empezar señalando, en primer lugar, la importancia del concepto de energía, uno de los más potentes, fructíferos y unificadores de la física clásica. Si a las razones anteriores añadimos su papel en otras ciencias, en la vida diaria, etc., se hace evidente la necesidad de introducirlo desde los primeros cursos de la enseñanza secundaria obligatoria. Por otra parte, la investigación educativa de las ideas previas de los alumnos sobre trabajo, energía y calor ha experimentado un gran desarrollo a partir de los años ochenta. También se ha originado una controversia, que todavía no se ha interrumpido, sobre la conveniencia de introducir el concepto de trabajo antes que el de energía o viceversa (Sexl, 1981; Duit, 1981; Warren, 1982). En un trabajo reciente (Tarín, 1996) se han analizados 38 artículos sobre el tema publicados en diversas revistas, algunas tan prestigiosas como Science Education, International Journal of Science Education, Enseñanza de las Ciencias, etc. porque, la importancia, tanto científica como tecnológica, del concepto de energía ha producido una notable investigación didáctica. Entre las dificultades detectadas podemos mencionar las siguientes: la confusión entre trabajo y esfuerzo (Driver y Warrington, 1985), considerar sólo uno de los factores que intervienen en el trabajo olvidando el otro, identificar trabajo y energía (Duit, 1984; Driver y Warrington, 1985), asignar un cierto carácter material a la energía (Duit, 1987a; Solomon, 1985), asociarla al movimiento, la actividad (Solomon, 1983) o a los procesos (Duit, 1984; Viglietta, 1990), considerar que la energía puede gastarse (Kesidou y Duit, 1993) o almacenarse (Solomon, 1985), dado que el lenguaje cotidiano está impregnado de expresiones como «consumo de energía», «crisis energética», etc., confundir las formas de energía con sus fuentes (Carr y Kirkwood, 1988; Solomon, 1985), atribuir la energía potencial al cuerpo y no a la interacción entre los cuerpos (Solbes y Martín, 1991), ignorar la variación de la energía interna (van Huls y van den Berg, 1993), asignar un carácter sustancial al calor (Albert, 1978; Erickson, 1979, 1980) o considerarlo como una forma de energía (von Roon, van Sparng y Verdonk, 1994), confundir la cantidad de calor y la temperatura 
(Arnold,1994). Y, muy en especial, no activar los esquemas de transformación, conservación, transferencia y degradación de la energía (Duit, 1981, 1984), sin los cuales no se puede comprender dicho concepto ni el principio de conservación.

Sin embargo, los trabajos anteriores no han resuelto todos los problemas; en particular, los siguientes:

- ¿Hasta que punto se tienen en cuenta en la enseñanza las ideas previas de los estudiantes, sus dificultades, y la necesidad de activar los esquemas de conservación, transformación, transferencia y degradación de la energía?

- ¿Se realiza un análisis crítico de las leyes de conservación clarificando si se trata de teoremas o de principios?

- ¿Se muestra el principio de conservación de la energía como un principio, no sólo de la mecánica y la termodinámica, sino de toda la física?

\section{HIPÓTESIS}

De acuerdo con lo anterior, se formula la siguiente hipótesis:

«El principio de conservación de la energía se introduce en la enseñanza de una forma poco clarificadora, sin tener en cuenta las dificultades de los alumnos y sin mostrar que es un principio de toda la física. En consecuencia, los estudiantes continúan utilizando sus preconceptos, no comprenden la conservación, transformación, transferencia y degradación de la energía; no tienen claro si la conservación de la energía es un principio o un teorema y no usan el principio de conservación de la energía en toda la física.»

Desarrollaremos con más detalle nuestra hipótesis en enunciados que sean contrastables:

En primer lugar, el tratamiento dado a la energía en mecánica activa, en los alumnos, los esquemas de transformación y de conservación de la energía. La transferencia y la degradación apenas se utilizan, a pesar de que los cuatro aspectos indicados se consideran necesarios para la comprensión del concepto de energía (Duit, 1981, 1984). Una prueba de ello es la escasa utilización de las cadenas de transformación y transferencia de energía. Por otra parte, los medios de comunicación transmiten una idea de conservación de la energía entendida como consumo, muy diferente de la que se considera en la ciencia. Es muy difícil entender el concepto de gasto de energía sin conocer su degradación (Duit, 1987b; Brook y Wells, 1988).

La conservación de la energía se plantea como un teorema deducido a partir de la segunda ley de Newton (y de la tercera, si se trata de un sistema de partículas). La secuencia seguida para la introducción de la conservación de la energía comienza por la definición de un trabajo puramente mecánico, entendido como el producto de la fuerza por la distancia que se desplaza su punto de aplicación. A continuación se deduce el teorema de las fuerzas vivas y se define la energía cinética y potencial. Por último, se obtiene la conservación de la energía imponiendo restricciones en el teorema de las fuerzas vivas. Llegados a este punto, algunos autores denominan principio de conservación de la energía al resultado obtenido.

Pensamos que esto constituye un error metodológico. Una expresión que se obtiene matemáticamente de un principio es un teorema. Si la conservación de la energía mecánica se ha derivado de las leyes de Newton, necesariamente tiene que ser contemplada como un teorema. De lo contrario, se olvida que, en la física, los nuevos principios son hipótesis verificadas por la experiencia y los experimentos conocidos hasta ese momento, y de los que se pueden deducir otras leyes. Sin embargo, este carácter es relativo, porque se puede tomar un teorema como principio, con lo cual el antiguo principio se convierte en teorema (por ejemplo, de la energía, en su formulación hamiltoniana, se deducen las leyes de Newton). La conservación de la energía tiene la categoría de principio universal que se cumple incluso en los casos en que la tercera ley de Newton no es válida (por ejemplo, en las interacciones electromagnéticas entre dos partículas cargadas en movimiento, cuya descripción hace necesaria la introducción de la energía y la cantidad de movimiento del campo).

En la expresión de la conservación de la energía mecánica no se especifica, en muchos casos, si el trabajo que aparece es conservativo, no conservativo, interno o externo. El trabajo de rozamiento se define operativamente pero no se relaciona con aspectos del calor o de energía interna. De esta forma, no se clarifican los límites de validez de la conservación de la energía mecánica ni se muestra la necesidad de un principio más general.

En pocas ocasiones se presenta un principio de conservación de la energía generalizado. Algunos textos y profesores lo hacen por medio de múltiples tipos de energía: mecánica, eléctrica, magnética, térmica, elástica, acústica, luminosa, química, etc. que se transforman entre sí. Otros, especialmente aquéllos que consideran la conservación de la energía en mecánica como un principio, consideran que todas las energías son reducibles a dos, la cinética y la potencial, incluyendo en ésta la gravitatoria, elástica, eléctrica (y, a veces, forzando el concepto de energía potencial, la magnética y la nuclear). La reducción de la energía a sólo dos clases representa un gran avance didáctico porque evita la multiplicidad de energías. Sin embargo, revela una concepción mecanicista de la física al olvidar la energía de los campos (y que éstos son, por tanto, entidades físicas tan reales como las fuentes que los crean), la de la masa en reposo y que la energía de interacción entre las partículas y los campos sólo puede ser denominada potencial si el campo es conservativo.

Sólo algunos libros de texto introducen el primer principio de la termodinámica presentándolo como el princi- 
pio de conservación de la energía. A pesar del avance que supone este principio, se enuncia de forma muy restringida, aplicable sólo a los sistemas termodinámicos (como, por ejemplo, un gas contenido en un cilindro con un émbolo y una fuente de calor). Con respecto a la mecánica, aparece la energía interna y el calor como un nuevo proceso de transferencia de energía al sistema debido a la diferencia de temperaturas. El trabajo que se utiliza es de tipo «presión multiplicada por variación de volumen. Desgraciadamente, no se suele relacionar con la conservación de la energía mecánica ni con los problemas mecánicos que implican disipación de calor. En este tema tampoco se analiza la degradación de la energía a través del segundo principio de la termodinámica.

Otra posibilidad, poco frecuente, utilizada en textos de nivel superior o artículos (Alonso y Finn, 1992), es el intento de deducir el primer principio de la termodinámica a partir de la energía propia de un sistema de partículas. De esta forma se comete el error metodológico antes mencionado (una expresión que se deduce de otra es un teorema) y se está dando una definición limitada, mecanicista, del calor, del trabajo y de la energía interna.

La elaboración del concepto de energía, hasta llegar a su total comprensión mediante el principio de conservación, fue un proceso de más de dos siglos de duración. Los enfoques antes mencionados son muy coherentes con las dos fases iniciales del desarrollo histórico de la ley de conservación:

- En la primera etapa, se establece la conservación de la energía en la mecánica. A partir del estudio de los choques elásticos (Huygens, Leibniz), se formula una ley de conservación en la que sólo aparece la energía cinética. Muy pronto se conocen las limitaciones de dicha ley. La conservación de la energía mecánica, no formulada claramente, es utilizada por Huygens para resolver el problema del péndulo y por Daniel Bernouilli en su estudio de los fluidos en movimiento. Hay que esperar casi medio siglo para que la conservación de la energía de un sistema mecánico sea establecida por Lagrange en su Mecánica analítica (1788). El carácter de teorema o de principio fundamental resulta confuso.

- En la segunda fase, se plantea la conservación de la energía en termodinámica. Se conceptualizan el calor, la cantidad de calor, la temperatura, la energía interna y el trabajo. Estos conceptos, unidos a una gran cantidad de hechos experimentales (procesos de conversión de energía, transformación de calor en trabajo; estudio de máquinas térmicas) dan lugar a la formulación de los dos principios de la termodinámica. El primer principio resuelve las limitaciones de la conservación de la energía en mecánica, mientras que el segundo aporta la degradación de la energía como un nuevo aspecto de la misma.

Este principio de conservación fue introducido por Mayer, Joule y Helmholtz con vocación de ser un principio de toda la física. Pero la física ha evolucionado mucho desde 1847 hasta la actualidad y es necesario tener en cuenta desarrollos históricos ulteriores.
En primer lugar, el establecimiento de la teoría electromagnética da lugar a la consideración de la energía de los campos y a la radiación como un nuevo proceso de transferencia de energía. La conservación de la energía en el electromagnetismo dice que la variación de la energía de los campos en una región del espacio (el sistema) es igual a la radiación (el flujo del vector de Poynting) más el trabajo realizado por los campos sobre las cargas y corrientes en el interior de dicha región. Como se puede ver, es un enunciado formalmente análogo al primer principio, si la energía interna incluyese la energía de los campos y se añadiera un nuevo término de radiación $\mathrm{R}$ al calor $\mathrm{Q}$ y el trabajo $\mathrm{W}$ (que debería generalizarse para incluir la polarización, imantación, etc.).

En segundo lugar, la energía en la física moderna. La teoría de la relatividad introduce la equivalencia de la masa y de la energía, y la energía de la masa en reposo, que es necesario incluir en el principio de conservación. La física cuántica muestra la cuantificación de la energía y su indeterminación (relación de Heisenberg energíatiempo). La desintegración puso en cuestión la conservación de la energía desde el punto de vista microscópico y el posterior descubrimiento del neutrino supuso su confirmación.

Por último, el teorema de Noether plantea una visión más profunda del sentido de la conservación de la energía al relacionarla con una invarianza de las leyes naturales bajo las traslaciones temporales.

\section{DISEÑO EXPERIMENTAL}

La contrastación de la hipótesis se ha realizado por medio de cuestionarios en los que se examina la enseñanza-aprendizaje de la energía desde la perspectiva de los libros de texto, profesores y alumnos.

\section{Cuestionario de libros de texto}

El cuestionario de libros de texto está formado por cinco apartados en los que se analizan aspectos de la energía relacionados con la mecánica, termodinámica, movimiento ondulatorio, electromagnetismo y física moderna. Los cuatro primeros han sido aplicados a libros de segundo y tercer cursos de BUP, así como a los de COU. También se han analizado textos correspondientes a los dos cursos de FP-1. Los ítems relacionados con la física moderna han sido revisados sólo en libros de COU.

Los cuestionarios de libros de texto, de profesores y de alumnos recogen ítems en los que se analizan aspectos semejantes de la energía, con el objeto de comprobar si existe coherencia entre los resultados obtenidos a través de instrumentos diferentes.

Dado el elevado número de ítems que forman el cuestionario de libros de texto, en la tabla IV.1 se presenta una selección de los mismos. 


\section{Cuestionario de profesores}

La elaboración del cuestionario dedicado a profesores, tanto en activo como en formación, se ha realizado con el objetivo de conocer la forma en que se enseña habitualmente el concepto de energía. Para ello, se han formulado tres preguntas relacionadas con dicho concepto (Tabla IV.2). El cuestionario consta de dos partes que se presentan por separado para evitar que los enunciados de unas cuestiones influyan en las respuestas de las restantes. La primera parte del cuestionario está formada por el ítem 1, en el que se pretende analizar varios aspectos de la enseñanza habitual de la energía, en concreto los siguientes:

a) ¿Se tienen en cuenta las ideas previas de los alumnos sobre trabajo, energía y calor?

b) ¿Se realizan actividades para desarrollar la transformación, conservación, transferencia y degradación de la energía?

c) ¿Se introduce la energía sólo en mecánica y en termodinámica?

Tabla IV.1

Resultados del cuestionario de libros de texto (selección).

\begin{tabular}{|c|c|c|c|}
\hline & $\begin{array}{l}\text { Nivel } 1 \\
\%\end{array}$ & $\begin{array}{c}\text { Nivel } 2 \\
\%\end{array}$ & $\begin{array}{c}\text { Global } \\
\%\end{array}$ \\
\hline \multicolumn{4}{|l|}{ 1.3. Se considera que los siguientes enunciados son principios de la física: } \\
\hline b) principio de conservación del momento angular & - & 21,4 & 21,4 \\
\hline c) principio de conservación de la energía & 38,5 & 53,3 & 46,4 \\
\hline \multicolumn{4}{|l|}{ 1.4. Se tienen en cuenta las ideas previas de los alumnos sobre: } \\
\hline a) trabajo & 26,7 & 16,7 & 21,2 \\
\hline b) energía & 13,3 & 11,1 & 12,1 \\
\hline c) calor & 13,3 & 5,9 & 9,4 \\
\hline \multicolumn{4}{|l|}{1.10 . } \\
\hline $\begin{array}{l}\text { a) Se introducen múltiples tipos de energía para generalizar la ley de } \\
\text { conservación de la energía }\end{array}$ & 61,5 & 18,8 & 37,9 \\
\hline b) Sólo se introduce la energía cinética y potencial & 38,5 & 81,3 & 62,1 \\
\hline 1.12. Se establecen los límites de validez de la conservación de la energía mecánica & 45,5 & 68,8 & 59,3 \\
\hline \multicolumn{4}{|l|}{ 1.13. Se activan los esquemas de: } \\
\hline a) transformación & 50,0 & 25,0 & 36,7 \\
\hline b) conservación & 78,6 & 100,0 & 90,0 \\
\hline c) transferencia & 28,6 & 31,3 & 30,0 \\
\hline d) degradación & 7,1 & 6,3 & 6,7 \\
\hline $\begin{array}{l}\text { 2.9. Se utilizan los principios de la termodinámica para explicar la crisis energética, } \\
\text { el calentamiento global, etc. }\end{array}$ & 11,1 & 0,0 & 7,7 \\
\hline 3.2. Se clarifica la transmisión de energía por las ondas y su carácter deslocalizado y continuo & 0,0 & 0,0 & 0,0 \\
\hline 4.3.a. Se indica que los campos eléctrico y magnético poseen energía & 0,0 & 22,2 & 12,1 \\
\hline $\begin{array}{l}\text { 4.3.b. Se indica que la energía del campo electromagnético es proporcional } \\
\text { al cuadrado de la amplitud del campo }\end{array}$ & 0,0 & 33,3 & 18,2 \\
\hline 5.3. Se explica el significado de la conservación de la masa/energía & - & 14,3 & 14,3 \\
\hline \multicolumn{4}{|l|}{$\begin{array}{l}\text { 5.4. Se explicita que las siguientes ecuaciones son consecuencia de la aplicación } \\
\text { del principio de conservación de la energía: }\end{array}$} \\
\hline a) ecuación del efecto fotoeléctrico & - & 0,0 & 0,0 \\
\hline b) ecuación de Bohr de las frecuencias del espectro del átomo de hidrógeno & - & 0,0 & 0,0 \\
\hline c) ecuación del efecto Compton & - & 76,9 & 76,9 \\
\hline
\end{tabular}




\section{Cuestionario de alumnos}

El cuestionario de alumnos ha sido redactado con el objeto de comprobar la existencia de ideas previas de los alumnos sobre el concepto de energía. También se pretende saber si conocen las limitaciones de la conservación de la energía en mecánica y termodinámica, y si utilizan el concepto de energía en partes de la física diferentes a las mencionadas.

El cuestionario consta de catorce preguntas. Los once primeros ítems se han aplicado a los alumnos del primer nivel ( $2^{\circ}$ de BUP y $4^{\circ}$ de ESO) mientras que los estudiantes del segundo nivel $\left(3^{\circ}\right.$ de BUP, COU y los dos cursos del bachillerato de Ciencias de la Naturaleza) han contestado todas las cuestiones. En la tabla IV.3, aparece una selección de los ítems.

\section{RESULTADOS}

Los resultados obtenidos en los cuestionarios de libros, de profesores y de alumnos se presentan y se analizan a continuación.

\section{Análisis de los resultados del cuestionario de libros de texto}

La tabla IV.1 recoge los resultados obtenidos al aplicar el cuestionario de libros de texto a una muestra formada por 16 libros del primer nivel y 18 del segundo.

Normalmente la conservación de la energía se obtiene en mecánica como un teorema, a pesar de que se trata de un principio universal, válido cuando no lo es la tercera ley de Newton. Este hecho se pone de manifiesto en los resultados del ítem 1.3. Muy pocos libros de texto consideran la conservación del momento angular, de la cantidad de movimiento o de la energía como principios generales de toda la física.

Las ideas previas de los alumnos sobre el trabajo, la energía o el calor son tenidas en cuenta por muy pocos libros de texto, en todos los niveles (ítem 1.4). Especialmente son ignoradas las que hacen referencia al calor $(9,4 \%)$.

Ítem 1.10. Se observa que la introducción de múltiples tipos de energía disminuye a medida que aumenta el nivel. De esta forma, frente a un $61,5 \%$ de textos del primer nivel que presenta varias clases de energía, se encuentra sólo un 18,8\% del segundo. Las energías no mecánicas que se citan mayoritariamente son la química, calorífica, térmica, magnética, luminosa y radiante. Dentro de la energía potencial figuran la gravitatoria, la elástica, la electrostática y la nuclear. Frente a la posición de considerar numerosas formas de energía, algunos libros presentan una postura reduccionista y mecanicista. Para éstos, todas las energías pertenecen a la clase de la energía cinética o potencial («sólo hay ener- gía mecánica que, a su vez, se divide en energía potencial y en energía cinética. Con frecuencia, se habla de energía térmica, energía eléctrica, energía química... pero todas estas energías no son más que formas de la energía mecánica»). Sin embargo, tal opinión no se puede considerar correcta porque olvida la energía de los campos libres y la de la masa en reposo.

Los límites de validez de la conservación de la energía en mecánica (ítem 1.12) aparecen en el 59,3\% de los libros de texto. Esta circunstancia hace que se presente la conservación de la energía como un teorema de la mecánica, sin ninguna conexión con otros dominios de la física.

De los cuatro aspectos fundamentales de la energía sólo la conservación es abordada por todos los textos analizados, excepto por el $25 \%$ de los libros de FP. Normalmente, las actividades relacionadas con la conservación están formadas por ejercicios cuantitativos en los que se plantea la conservación de la energía mecánica en dos o más puntos de una trayectoria con el objeto de calcular el valor de una magnitud. Algunos libros señalan el carácter de artificio matemático para dicho procedimiento como una forma más sencilla de resolución de problemas ( $«$ Se introducen el concepto de trabajo y energía porque se pueden resolver problemas...»). Muy pocas veces aparecen actividades cualitativas de conservación. La transformación de la energía suele presentarse únicamente en los problemas de conservación pero no se pregunta explícitamente por la energía transformada. Tampoco se realizan cadenas de conversión ni se analizan gráficas. Por otra parte, se observa que el número de textos que proponen actividades de transformación disminuye en los niveles superiores. Una situación análoga se presenta con la transferencia de energía, que aparece en situaciones cuantitativas relacionadas generalmente con el teorema de las fuerzas vivas. Sin embargo, la degradación de la energía sólo se desarrolla en el 6,7\% de los libros a pesar de la realización de problemas de conservación de energía relacionados con el rozamiento. Éstos suelen explicarse, de una manera superficial, como la transformación de una parte de la energía en calor. De las consideraciones anteriores se puede concluir que la enseñanza de la energía a través de los libros se basa esencialmente en la conservación y, en menor medida, en la transformación. Los procesos de transferencia y la degradación apenas se muestran.

La utilización de la degradación de la energía en la explicación de fenómenos de la vida diaria como la crisis energética o el calentamiento global (ítem 2.9) aparece como irrelevante en los libros. El primer fenómeno es recogido sólo en un libro de $2^{\circ}$ de BUP, mientras que el segundo no aparece en ninguno. Este resultado pone de manifiesto la imagen de la ciencia que presentan algunos libros. Se trata de una ciencia sin ninguna conexión con la vida diaria, que presta escasa atención al análisis de fenómenos cotidianos.

Ítem 3.2. Aunque el concepto de intensidad de las ondas aparece recogido prácticamente en la totalidad de los libros, sólo el 43,8\% lo relaciona con el cuadrado de la 
amplitud. Este resultado confirma la hipótesis de que la enseñanza de la energía se reduce a la cinética, a la potencial y, en algunos casos, a la interna pero no se considera en otros campos de la física.

Ítem 4.3. Sólo el 12,1\% de los textos señala que el campo eléctrico y el magnético poseen energía. Normalmente, la energía del primero aparece citada cuando se examina un condensador cargado. Sin embargo, ningún libro de FP-1 ni de $2^{\circ}$ o $3^{\circ}$ de BUP se refiere a la energía del campo magnético, mientras que únicamente cuatro textos de COU mencionan dicha energía. Consecuentemente con las consideraciones anteriores, en un número muy reducido de textos $(19,4 \%)$ se muestra la expresión cuantitativa de la energía del campo electromagnético.

Como consecuencia de una falsa interpretación de la equivalencia masa/energía, se producen confusiones en la conservación de ambos conceptos. En algunos libros, se niega la conservación de la masa en términos como los siguientes: «No se conserva, sin embargo, la masa, ya que en toda reacción nuclear la suma de la masa de los reaccionantes no coincide con la suma de las masas de los productos». Según otros libros, la energía no se mantiene constante: «Debido al defecto de masa existente en el núcleo, en las reacciones nucleares no se cumple la ley de conservación de la energía, por lo que aparece en la reacción cierta cantidad de energía.» En otras ocasiones, se indica que se incumple tanto la conservación de la masa como la de la energía. De todo lo anteriormente expuesto se deduce claramente la confusión que existe cuando se trata de aplicar los principios de conservación de la masa y de la energía a las reacciones nucleares. Seguramente la causa de este problema se encuentra en que muy pocos libros $(26,7 \%)$ señalan que la equivalencia de la masa y la energía supone también la equivalencia entre los principios de conservación de los dos conceptos (ítem 5.3).

Ítem 5.4. En ningún libro de los analizados se señala que la ecuación del efecto fotoeléctrico es una consecuencia de la aplicación de la conservación de la energía. Lo mismo sucede con la ecuación de las frecuencias del espectro del átomo de hidrógeno, según la interpretación de Bohr. Sin embargo, un 76,9\% de los textos indica la conservación de la cantidad de movimiento y de la energía en el efecto Compton. De nuevo se comprueba que la conservación de la energía no es utilizada por los libros como vínculo unificador de todas las ramas de la física.

\section{Análisis de los resultados del cuestionario de profesores}

La opinión de los profesores sobre la enseñanza de la energía se ha analizado a través de la interpretación de las respuestas al cuestionario que han contestado 38 profesores en formación (alumnos del curso de Aptitud Pedagógica) y 22 profesores en activo, pertenecientes a varios institutos.

Tabla IV.2

Resultados del cuestionario de profesores.

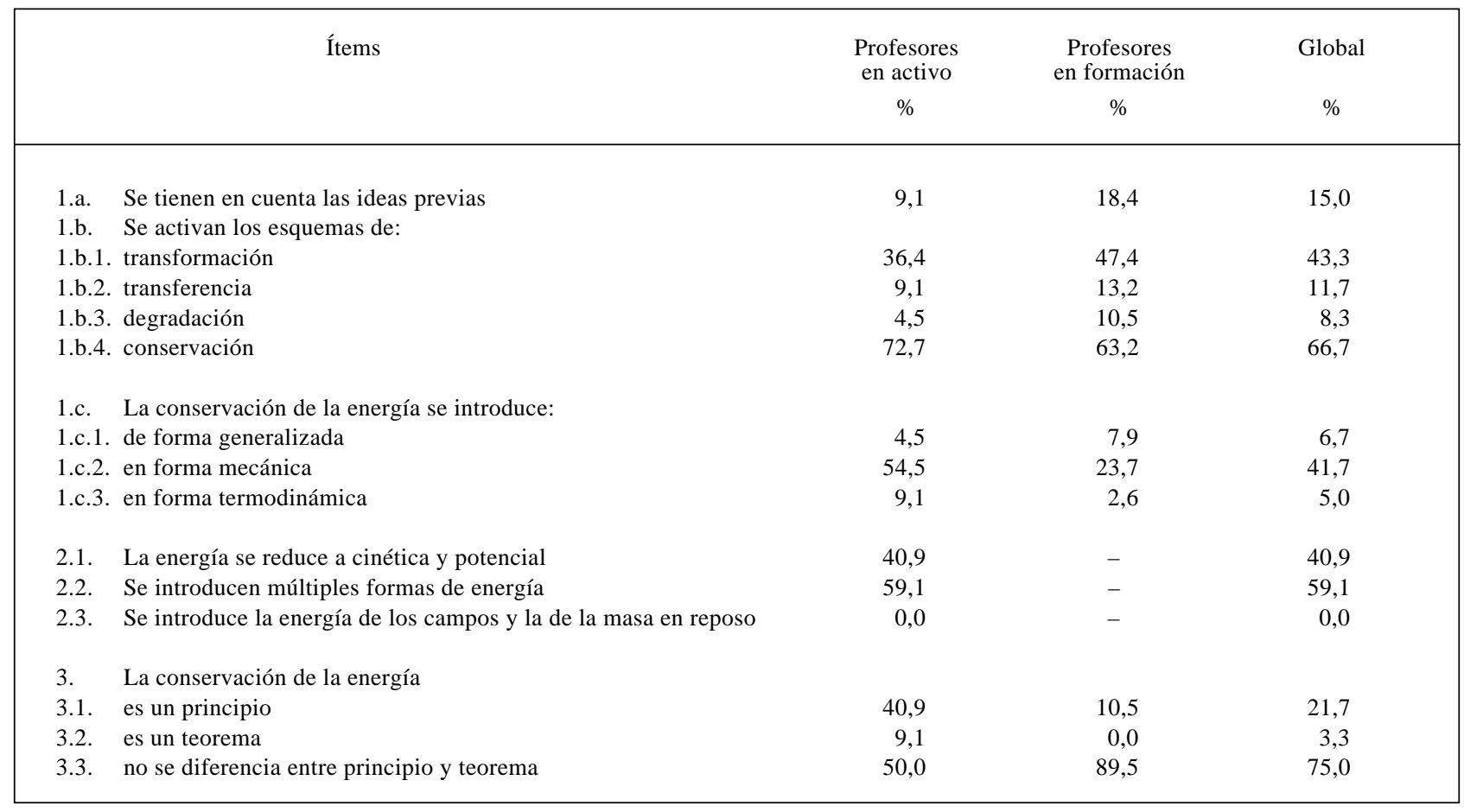


En la tabla IV.2. se muestran los resultados obtenidos en el cuestionario, expresados como porcentajes (\%) de respuestas afirmativas a los enunciados que aparecen en dicha tabla.

Resulta muy reducido el porcentaje de profesores, tanto en activo $(9,1 \%)$ como en formación $(18,4 \%)$, que creen conveniente considerar las ideas previas de los alumnos al introducir el concepto de energía (ítem 1).

De los cuatro aspectos fundamentales de dicho concepto, la conservación aparece, para los profesores en activo y en formación, como la más importante $(72,7 \%$ y $63,2 \%$ respectivamente). La transformación es introducida por el $36,4 \%$ y $47,4 \%$ de los profesores. Sin embargo, la transferencia es considerada por un número menor $(9,1 \%$ y $13,2 \%)$, mientras que un porcentaje todavía más reducido $(4,5 \%$ y $10,5 \%)$ propone la degradación de la energía en la introducción de dicho concepto.

En correlación con los resultados del análisis de textos, las cifras anteriores ponen de manifiesto que la energía se enseña fundamentalmente a través de su conservación y transformación. La transferencia se tiene en cuenta en menor media y la degradación en un grado todavía más pequeño. Las consecuencias de este tipo de enseñanza se manifiestan en una visión de la energía alejada completamente de los fenómenos cotidianos como el consumo de combustibles, la crisis energética, etc., imposibles de explicar por medio de la conservación.

Un reducido número de profesores en activo y en formación $(13,6 \%$ y $36,8 \%$ respectivamente) propone una enseñanza de la energía de una forma general, partiendo de unas definiciones no mecánicas de energía y trabajo. La relación entre los dos conceptos se realiza por medio de la relación $\mathrm{W}=\Delta \mathrm{E}$.

Resulta elevado el número de profesores en activo (60\%) que, de acuerdo con nuestra hipótesis, introduce la conservación de la energía exclusivamente en mecánica. Contrariamente, sólo el 23,7\% de los profesores en formación presentaría la energía exclusivamente desde la mecánica. Dicha enseñanza es realizada por los procedimientos tradicionales: definición de trabajo, energía cinética y potencial, deducción del teorema de las fuerzas vivas, aplicación de dicho teorema a fuerzas conservativas $\mathrm{y}$, finalmente, a las no conservativas (rozamiento).

La introducción de la energía en termodinámica es aceptada por el $9,1 \%$ de los profesores en activo y por un porcentaje menor en el caso de los profesores en formación. En este caso, se realiza introduciendo el concepto de energía interna y el de calor como transferencia de energía, formulando la conservación como $\Delta \mathrm{E}_{\text {sistema }}=\mathrm{Q}+\mathrm{W}$.

En el ítem 2 se espera encontrar, de acuerdo con nuestra hipótesis, que los profesores presenten una visión limitada de las clases de energía. En un caso aparecerán múltiples formas de energía y, en otros, se reducirán a la cinética y potencial. Los resultados indican que el 40,9\% de los profesores en activo piensan que toda la energía puede reducirse a cinética y potencial, sin considerar la energía de los campos y de la masa en reposo. Por otra parte, algunos profesores indican varias formas de energía como la nuclear, calorífica, interna, química, del sonido, solar, eólica, motora o calorífica. Sólo tres profesores hacen referencia a la energía del campo electromagnético.

La mitad de los profesores en activo y el $89,5 \%$ de los profesores en formación encuestados muestran dificultades en diferenciar un principio de un teorema (ítem 3). Con referencia a la conservación de la energía, algunos profesores del primer grupo señalan que «es un principio pero si se comprueba pasa a ser teorema», que «si la energía se conserva es principio y si se transforma, teorema», etc.

Para el 52,6\% de los profesores en formación, un enunciado se puede determinar como principio o teorema en base a su demostrabilidad, como se pone de manifiesto en las siguientes opiniones: «un principio es algo que se enuncia pero que resulta difícil de demostrar, mientras que un teorema es demostrable», «un principio es lo que tomamos como un enunciado no experimentado», «los teoremas son el resultado de una experiencia», «un teorema es una teoría comprobada», «un teorema se cumple siempre y ha sido demostrado a partir de la experiencia». En otros casos $(13,2 \%)$, la diferencia entre principio y teorema se encuentra en su grado de cumplimiento: «un teorema se cumple siempre y un principio, no», «un teorema siempre se cumple, un principio es una teoría». También se asocian los teoremas a una deducción matemática: «un principio no requiere un desarrollo matemático». Por último, un profesor en formación indica que «un teorema es una relación entre dos o más magnitudes, mientras que un principio nos dice que existe una relación entre dos o más magnitudes sin decir cómo están relacionadas».

La conservación de la energía es considerada como principio por el 40,9\% de los profesores en activo y por el $10,5 \%$ de los profesores en formación, mientras que ninguno de éstos reconoce dicha conservación como teorema, frente a un $9,1 \%$ de aquéllos. Resulta destacable señalar que ninguna respuesta de ambos grupos hace referencia al carácter general de la conservación de la energía, aplicable a todos los campos de la física.

\section{Análisis de los resultados del cuestionario de alumnos}

Los resultados obtenidos en una selección de los ítems del cuestionario de alumnos se recogen en la tabla IV.3. En ella aparece el porcentaje de respuestas correctas, y no favorables a nuestra hipótesis, correspondientes a cada uno de los ítems.

La muestra de alumnos está formada por un total de 320 , pertenecientes a dos institutos de BUP y uno de secundaria, situados en Valencia y su provincia. Los resultados se han clasificado en dos niveles. El primero $(\mathrm{N}=152)$ comprende los grupos de $2^{\circ}$ de BUP y de $4^{\circ}$ de 
ESO, y el segundo $(\mathrm{N}=168)$ los de $3^{\circ}$ de BUP, COU y $1^{\circ}$ y $2^{\circ}$ del bachillerato de Ciencias de la Naturaleza. En la tabla de resultados aparecen como nivel 1 y nivel 2 .

Ítem 2. El 15\% de alumnos del nivel 1 y el 33,6\% del nivel 2 reconocen la energía como una propiedad de todos los cuerpos. En este caso, se señala la energía interna como la común a todos los sistemas pero no se menciona la energía de la masa en reposo. Algunos alumnos indican que el fotón y el neutrino no tienen energía y, en algún caso muy aislado, que todos los sistemas con masa tienen energía. Generalmente, se mencionan como sistemas sin energía los que se encuentran en reposo o «los que están libres de influencias externas».

Ítem 3. Aunque la energía se conserva en todos los fenómenos, los alumnos citan mayoritariamente los de tipo mecánico y, en muy escasas ocasiones, los termodinámicos. Sólo el 20,3\% de los estudiantes señala procesos de otros campos de la física. Esta característica tiene seguramente su origen en la introducción de la energía en la enseñanza a través de la mecánica y la termodinámica. Los fenómenos que indican los alumnos se relacionan con cuerpos que ascienden, caen o chocan, muelles, etc. Como ejemplos de fenómenos termodinámicos aparecen cuerpos que se calientan o enfrían. En algunas ocasiones, los alumnos no diferencian los fenómenos de las situaciones estáticas como «la presión que soporta un submarinista dentro del agua». Entre los fenómenos no mecánicos, los alumnos hacen referencia a la combustión, la conversión de la energía del sol en energía eléctrica realizada en una placa solar o la transformación de la energía cinética del viento en eléctrica.

Ítem 4. Los alumnos muestran dificultades para analizar el fenómeno considerando el sistema pelota-suelo. Casi todos se centran en examinar, de manera exclusiva, las variaciones de la energía de la pelota. De esta forma, se reconoce que dicha energía disminuye porque cada vez bota menos o porque pierde «fuerza». Como causas de esa variación se indica que, en cada salto, la pelota «debe superar el rozamiento y la gravedad», ésta «le quita fuerza» o parte de la energía se queda en el suelo. En

Tabla IV.3

Resultados del cuestionario de alumnos (selección).




otros casos, se considera que la energía de la pelota se conserva y, al mismo tiempo, se va perdiendo porque «la energía tiene menos fuerza». También se señala que la energía deja de conservarse en el instante en el que la pelota se para porque, en ese momento, su energía es cero. Muy pocos alumnos del nivel 1 y 2 relacionan la transformación de la energía mecánica con la energía interna o con el calor. El ítem ha sido contestado correctamente por el $6,4 \%$ de los alumnos.

Las respuestas del ítem 5 se han agrupado en tres apartados. En el primero figuran las que indican que la conservación de la energía es un teorema, mientras que el segundo recoge las respuestas que consideran dicha conservación como principio. El tercer grupo lo forman las respuestas que justifican su elección en base a una diferenciación incorrecta entre principio y teorema. El $6,3 \%$ de los alumnos piensa que la conservación de la energía es un teorema frente a un porcentaje mayor $(22,5 \%)$, que la considera como principio. Sin embargo, la mayoría de los estudiantes $(71,3 \%)$, en correlación con los resultados del cuestionario de profesores, tiene dificultades en explicar correctamente la diferencia entre un principio y un teorema, como se pone de manifiesto en estos ejemplos: «los teoremas se demuestran, como el de Pitágoras, pero los principios, no», «si demuestro un principio, ya no es principio y se convierte en teorema».

Ítem 7. Casi todos los alumnos $(98,7 \%)$ reconocen que existen muchas formas de energía. Las más citadas se relacionan con la mecánica, el calor, la electricidad o las nuevas energías. Entre ellas, aparecen la energía gravitatoria, elástica, mecánica, térmica, calorífica, eléctrica, solar y eólica. También se hace referencia a otros tipos de «energías mecánicas» como la hidráulica, «cinestésica», aerostática, hidrostática, centrífuga y estática. Por último, se cita la energía geotérmica, de la biomasa, magnética, de los combustibles, radiactiva, nuclear y fotolítica. Muy pocos alumnos indican que la energía es sólo cinética y potencial. Alguno señala que toda la energía se puede reducir a cinética porque no existe la potencial. En muy pocas respuestas aparece la energía electromagnética y en ninguna la de la masa en reposo.

Ítem 8. Según la opinión casi unánime de los alumnos $(98,9 \%)$, la energía potencial se atribuye al cuerpo situado en un campo gravitatorio. En algunos casos, se detalla que dicha energía se encuentra en la parte inferior del cuerpo, en su centro de gravedad o en la velocidad. Para un número muy reducido de alumnos la energía potencial «está en el cuerpo pero pasa a la altura». También se señala que la energía potencial se encuentra en la altura, en la gravedad, en el peso del cuerpo, en «la fuerza con la que cae», en «el trabajo hecho para subirlo», en «la atracción de la Tierra»o «entre el peso y la altura».

Ítem 9. Muy pocos alumnos $(32,9 \%)$ analizan correctamente el fenómeno de enfriamiento de un metal. Tal como sucede en el ítem 4, la mayoría de los estudiantes interpreta el proceso considerando sólo el trozo de hierro, sin utilizar el concepto de sistema. De esta forma, señalan que la energía del hierro disminuye y no se conserva, pero no indican ningún proceso de transferencia de dicha energía al aire. También se constatan las dificultades que presenta el concepto de calor. Una mayoría de alumnos confunden el calor, como proceso de transferencia de energía, con una clase de energía. Ésta es llamada, en algunas ocasiones, energía calorífi$c a$, y se encuentra en el interior de los cuerpos. De acuerdo con esto, se dice que «la energía del hierro se conserva pero el calor vale cero porque se enfría»o «la energía calorífica del hierro se pierde pero la energía interna se conserva». Aunque un número muy reducido de alumnos (1\%) menciona los mecanismos de transferencia de energía, algunos señalan, de una forma muy implícita, una posible transferencia al indicar que «el hierro desprende energía» o «el hierro pierde calor». Cuando se analiza el fenómeno teniendo en cuenta el sistema, se utilizan de forma incorrecta ciertos conceptos. De esta manera, se dice que la energía se conserva en el sistema porque «está aislado y no entra ni sale calor», sin considerar las transformaciones de energía que han ocurrido en el interior del sistema.

Ítem 10. Muchas respuestas describen la serie de fenómenos que tienen lugar en el proceso pero no indican las transformaciones de energía que se producen. La construcción de la cadena de transformaciones ha sido realizada correctamente por el $10,5 \%$ de los estudiantes. En este ítem reaparece la idea de multiplicidad de energías que tienen los alumnos. De esta forma, se hace referencia a la energía «de las aguas», hidráulica, calorífica o térmica, e incluso al calor como forma de energía. En cambio, la energía interna aparece muy pocas veces, incluso en el nivel superior. Los resultados anteriores ponen de manifiesto la escasa activación del esquema de transformación de energía en los alumnos.

Ítem 11. La mayoría de los alumnos relaciona la crisis energética con el uso de la energía, la cual no se conserva porque se utiliza, se gasta y se agota. Algunos alumnos piensan que la energía es difícil de conservar o de recuperar. Otros creen que no se conserva porque se transforma. En algunas respuestas se admite parcialmente la conservación de la energía, en el sentido de que «existen energías que se conservan y otras, no» o «la energía se conserva "hasta cierto punto"». Muy pocos alumnos $(16,5 \%)$ señalan la degradación de la energía como explicación de la crisis. Las ideas mostradas por los alumnos en las respuestas de este ítem reflejan el sentido de la conservación de la energía en el lenguaje cotidiano. Mientras que la física señala que la energía se conserva, la experiencia de la vida diaria parece estar en contra de la afirmación científica porque la energía se gasta (Duit, 1981).

Ítem 12. Un número muy reducido de respuestas $(13,1 \%)$ recoge la idea de la transmisión de energía por medio de las ondas electromagnéticas desde la emisora al receptor de televisión. Para muchos alumnos, las ondas se transforman en imagen y sonido al alcanzar el televisor, sin especificar cómo lo hacen. Algunos estudiantes explican la formación de la imagen en el televisor por medio de los electrones pero no se refieren a las ondas electromagnéticas. Aunque, en algunos casos, se señala la transmi- 
sión de impulsos, códigos u órdenes por medio de las ondas electromagnéticas, no se relaciona con la energía. En términos generales, las respuestas señalan que pocos alumnos reconocen la existencia de la energía del campo electromagnético y su transmisión por radiación, incluso en fenómenos tan cotidianos como las emisiones de televisión.

\section{CONCLUSIONES}

Los resultados obtenidos permiten señalar que los libros de texto y los profesores:

1. No tienen en cuenta las ideas previas de los alumnos y no clarifican los principios y los teoremas de la mecánica. Por otra parte, presentan la conservación de la energía en mecánica y en termodinámica; en el primer caso, como un teorema y, en el segundo, como una generalización de situaciones particulares. En ninguna de las dos situaciones se generaliza la conservación de la energía.

2. Realizan la enseñanza de la energía fundamentalmente a través de su conservación y, en menor grado, por medio de su transformación. Sin embargo, la transferencia y la degradación aparecen muy pocas veces. Estos aspectos fundamentales del concepto de energía se utilizan raramente para explicar fenómenos cotidianos, no abordables considerando únicamente la conservación.

3. En raras ocasiones muestran que la conservación de la energía es un principio de toda la física. Como consecuencia, no utilizan dicho principio para explicar fenómenos de otros campos, como el electromagnetismo, las ondas o la física moderna. Tampoco se citan las contribuciones de dichos dominios: la energía del campo libre o de la masa en reposo, y la equivalencia masa/energía. No se hace referencia a los problemas que aparecieron en dichos campos con respecto a la conservación de la energía.

Como consecuencia de la enseñanza que se realiza del concepto de energía, se puede señalar que los alumnos:

1. Siguen manteniendo ideas equivocadas sobre la energía. Así, asocian la energía al movimiento, localizan la energía potencial en el cuerpo, creen que la energía se gasta, etc.

2. No utilizan espontáneamente la energía ni su transformación, conservación, transferencia y degradación para interpretar cualquier clase de fenómenos físicos, incluidos los mecánicos.

3. Piensan que la conservación de la energía es una ley que se utiliza en la mecánica y en la termodinámica pero no reconocen que se cumple en todos los dominios de la física y no diferencian si se trata de un principio o de un teorema.

\section{PERSPECTIVAS. UNA PROPUESTA}

Estos resultados, así como los obtenidos en los y las estudiantes (Tarín, 1996) ponen de manifiesto la necesidad de elaborar una propuesta y unos materiales alternativos para la enseñanza de la energía, propuesta que deberá ser discutida con otros profesores y utilizada con estudiantes, cuyos resultados habrán de ser comparados con los de los estudiantes a los que se les ha impartido la enseñanza usual de la conservación de la energía.

La propuesta consistiría básicamente en seguir el desarollo histórico del concepto de energía, mostrando sus limitaciones, e incluyendo los nuevos aspectos que han aumentado la potencia del concepto. Por ello, se podría comenzar con la idea de energía como «capacidad de un cuerpo para transformar la materia» ya que parece surgir sin dificultades aparentes. Algunos autores han señalado que esta idea no es correcta porque todos los sistemas tienen energía y, sin embargo, no todos pueden realizar trabajo (por ejemplo, muchos sistemas tienen sólo energía interna pero, como están a la misma temperatura que el exterior, no pueden producir transformaciones. Esto les ha llevado a definir la energía como una propiedad de los sistemas que sólo cambia cuando se producen interacciones, lo cual es cierto, pero no suficiente, porque esto se puede afirmar de otras magnitudes que se conservan en los sistemas: la cantidad de movimiento, el momento angular, etc. Mejorar esto afirmando que la energía es la magnitud que se conserva debido a la invarianza de las leyes de la física respecto a traslaciones temporales nos llevaría demasiado lejos.

A continuación se introduciría la ley de conservación de la energía mecánica, mostrando sus limitaciones, que harían necesario introducir el primer principio de la termodinámica. Pero, en el nivel de primer curso de bachillerato, sería conveniente señalar como la introducción del concepto de campo electromagnético por Faraday y Maxwell y su confirmación experimental por Hertz, y la teoría especial de la relatividad de Einstein, que se estudiarán en el segundo curso, han producido nuevos problemas en la energía y su conservación.

Todo este proceso va acostumbrando al estudiante a pensar en la energía como una magnitud que se conservaba de forma general y permite introducir un principio de conservación de la energía general: la energía total de un sistema aislado se conserva. Por sistema aislado o cerrado entendemos aquél en que no hay intercambios o transferencias de energía con su entorno mediante trabajo, calor, ondas mecánicas o electromagnéticas o cualquier otro proceso de transferencia.

Para determinar la energía total hay que identificar los tipos de energía del sistema. En general, podemos encontrarnos con energía de las partículas libres (la cinética y la de su masa en reposo), energía de los campos libres, energía de las interacciones entre partículas y campos (que es potencial si los campos son conservativos). Cada una de estas contribuciones a la energía 
total del sistema puede variar con el tiempo transformándose en una de otro tipo, pero su suma no cambia, se conserva.

Sin embargo, con las propiedades de transformación, conservación y transferencia no está completa nuestra descripción de la energía. Para ello es necesario que el estudiante comprenda que en la naturaleza hay procesos irreversibles, los cuales, si sólo existiese el principio de conservación de la energía, se podrían verificar en ambos sentidos, pero en realidad evolucionan en un sólo sentido, debido a la degradación de la energía.

En segundo curso de bachillerato hay que aprovechar todas las ocasiones para utilizar la ley general de conservación de la energía, no sólo en el consabido estudio energético de la interacción gravitatoria y eléctrica. También es necesario mostrar la transferencia de energía mediante ondas mecánicas y electromagnéticas, energía

\section{REFERENCIAS BIBLIOGRÁFICAS}

ALONSO, M. y FINN, E.J. (1992). Física. Madrid: Addison Wesley.

ARNOLD, M. (1994) Children's and lay adults' views about thermal equilibrium. International Journal of Science Education, 16(4), pp. 405-419.

BROOK, A. y WELLS, P. (1988). Conserving the circus? Physics Education, 23, pp. 80-85.

CARR, M. y KIRKWOOD, V. (1988). Teaching and learning about energy in New Zealand secondary school junior science classrooms. Physics Education, 23, pp. 87-91.

DRIVER, R. y WARRINGTON, L. (1985). Students' use of the principle of energy conservation in problem situations. Physics Education, 20, pp. 171-176.

DUIT, R. (1981). Understanding Energy as a Conserved QuantityRemarks on the Article by R.U. Sexl. European Journal of Science Education, 3(3), pp. 291-301.

DUIT, R. (1984). Learning the energy concept in schoolempirical results from The Philippines and West Germany. Physics Education, 19, pp. 59-66.

DUIT, R. (1987a). Should energy be illustrated as something quasi-material? European Journal of Science Education, 9(2), pp. 139-145.

DUIT, R. (1987b). Der 2. Hauptsatz der Thermodynamik-ein Thema für alle Schulstufen. Naturwissenschaften im UnterrichtPhysik/Chemie, 35(24), pp. 140-146.

ERICKSON, G. (1979). Children's conceptions of heat and temperature. Science Education, 63(2), pp. 221-230.

ERICKSON, G. (1980). Children's viewpoints of heat: A second look. Science Education, 64(3), pp. 323-336. continua y deslocalizada en el frente de ondas, cuyo valor es proporcional al cuadrado de la amplitud (de la onda mecánica, del campo electromagnético): en relatividad, introducir la energía en reposo y la equivalencia entre masa y energía, aplicándola a la radiactividad y las reacciones nucleares; en cuántica, mostrar el carácter cuantizado de la energía de la radiación y los sistemas atómicos, aplicando el principio de conservación al efecto fotoeléctrico y las transiciones atómicas. La proporcionalidad de la intensidad de la onda con el cuadrado de la amplitud permite justificar la interpretación probabilista de Born. Se puede aplicar la relación de indeterminación de Heisenberg energía-tiempo para estimar la anchura de las rayas espectrales o la masa de la partícula intercambiada en las interacciones. Por último, conviene mostrar cómo la desintegración puso en cuestión la conservación de la energía desde el punto de vista microscópico y el posterior descubrimiento del neutrino supuso su confirmación.

MICHINEL, J.L y D'ALESSANDRO, A. (1994). El concepto de energía: de las concepciones previas a la propuestas de un nuevo sublenguaje. Enseñanza de las Ciencias, 12(3), pp. 369-381.

SEXL, R.U. (1981). Some Observations Concerning the Teaching of the Energy Concept. European Journal of Science Education, 3(3), pp. 285-289.

SOLBES, J. y MARTÍN, J. (1991). Análisis de la introducción del concepto de campo. Revista Española de Física, 5, pp. $34-40$.

SOLOMON, J. (1983). Learning about energy: how pupils think in two domains. European Journal of Science Education, 5(1), pp. 49-59.

SOLOMON, J. (1984). Alternative views of energy. Physics Education, 19, p. 56.

SOLOMON, J. (1985). Teaching the conservation of energy. Physics Education, 20, pp. 165-176.

TARÍN, F. (1996). Enseñanza-aprendizaje de la energía. Memoria de investigación (no publicada).

VAN HULS, C. y VAN DEN BERG (1993).Teaching energy: a systems approach. Physics Education, 28, pp. 146-153.

VIGLIETTA, L. (1990). A more «efficient» approach to energy teaching. International Journal of Science Education, 12(5), pp. 491-500.

VON ROON, P.H., VAN SPRANG, H.F. y VERDONK, A.H. (1994). Work and Heat: on a road towards thermodynamics. International Journal of Science Education, 16(2), pp. 131-144.

WARREN, J.W. (1982). The nature of energy.European Journal of Science Education, 4(3), pp. 295-297. 